\title{
ФУНКЦИОНАЛЬНЫЕ ПРЕДСТАВЛЕНИЯ РЕШЕТОЧНО УПОРЯДОЧЕННЫХ ПОЛУКОЛЕЦ. ІІІ
}

\author{
В. В. Чермных, О. В. Чермных
}

\begin{abstract}
В статье рассматриваются решеточно-упорядоченные полукольца ( $d r l$-полукольца). Определены компактные пучки $d r l$-полуколец, получена их характеризация. Изучаются свойства компактных пучков. В частности, описано строение неприводимых и максимальных $l$-идеалов $d r l$-полукольца сечений компактного пучка. Получено описание компактного пучка функциональных полуколец ( $f$-полуколец) в терминах непрерывного отображения его неприводимого (и максимального) спектра на компакт. В статье также содержится доказательство того, что $f$-полукольцо является гельфандовым тогда и только тогда, когда оно изоморфно полукольцу всех сечений компактного пучка $f$-полуколец с единственным максимальным идеалом.
\end{abstract}

Ключевые слова: решеточно упорядоченное полукольцо, функциональное полукольцо, компактный пучок, гельфандово $f$-полукольцо. III.

V. V. Chermnykh, O. V. Chermnykh. Functional representations of lattice-ordered semirings.

Lattice-ordered semirings ( $d r l$-semirings) are considered. Compact sheaves of $d r l$-semirings are defined and their characterization is obtained. The properties of compact sheaves are studied; in particular, the structure of irreducible and maximal $l$-ideals in the $d r l$-semiring of sections of a compact sheaf is described. A compact sheaf of functional semirings ( $f$-semirings) is described in terms of a continuous mapping of the irreducible (and maximal) spectrum of this sheaf onto a compact Hausdorff space. The paper also contains a proof that an $f$-semiring is Gelfand if and only if it is isomorphic to the semiring of all sections of a compact sheaf of $f$-semirings with a unique maximal ideal.

Keywords: lattice-ordered semiring, functional semiring, compact sheaf, Gelfand $f$-semiring.

MSC: $16 \mathrm{Y} 60$

DOI: $10.21538 / 0134-4889-2020-26-3-235-248$

\section{Введение}

Изучаемые в статье $d r l$-полукольца образуют широкий класс решеточно упорядоченных полуколец. Впервые $d r l$-полукольцо было рассмотрено в [1], и его определение базировалось на понятии $d r l$-полугруппы, введенном в обиход в [2]. Подробно алгебраические свойства $d r l$ полуколец изучались в [3].

В статьях [4;5] было построено несколько пучков $d r l$-полуколец и были получены представления $d r l$-полуколец сечениями этих пучков. Данная работа является продолжением указанных статей.

В качестве основных объектов исследования мы рассматриваем компактный пучок $d r l$-полуколец и компактные представления - изоморфные представления $d r l$-полуколец сечениями компактных пучков. Понятие компактных пучков алгебр впервые было введено в научный оборот при изучении пирсовских пучков колец [6], при представлениях строго гармонических и гельфандовых колец [7;8], нормальных решеток [9] и затем - строго гармонических положительных [10] и гельфандовых полуколец [11]. Компактные пучки обладают накрывающими пространствами с хорошими свойствами "отделимости сечениями". Встречаются компактные пучки достаточно часто: кроме ламбековсих пучков указанных выше алгебр к компактным пучкам относятся пирсовские пучки колец и полуколец, пучки над нульмерными компактами. 
Их широкое распространение привело к созданию серии работ, посвященных общей теории компактных пучков и компактных представлений [11-13].

Среди пучков $d r l$-полуколец (см. [4;5]) также нашлись пучки с хорошей Г-отделимостью в накрывающем пространстве. Это является первой причиной для определения компактных пучков $d r l$-полуколец и изучения их свойств. Вторая причина - желание выстроить общие конструкции, под которые попадали бы уже имеющиеся пучки и пучковые представления. И наконец, имеющиеся наработки о компактных пучках $f$-полуколец позволили получить новый результат о пучковой характеризации гельфандова $f$-полукольца.

Укажем основные результаты статьи. Нами дается характеризация компактных пучков (предложение 1). Заметим, что теория пучковых представлений абстрактных (т. е. не обязательно упорядоченных) полуколец развита к настоящему времени достаточно хорошо. Поэтому возникает естественный вопрос: какие результаты общей теории функциональных представлений полуколец могут использоваться при изучении представлений $d r l$-полуколец? Предлагаемая работа может считаться первой попыткой ответа на поставленный вопрос. А именно, пусть $(\Pi, X)$ - компактный пучок $d r l$-полуколец, $Г(П)-d r l$-полукольцо всех глобальных сечений этого пучка, $J_{x}-l$-идеал всех глобальных сечений, равных нулю в точке $x$. Одним из свойств в теореме 1 является условие

$$
J_{x}+J_{y}=\Gamma(\Pi) \quad \text { для различных } \quad x, y \in X .
$$

Аддитивная формулировка этого свойства позволяет для исследования компактных пучков $d r l$-полуколец опираться на полукольцевые результаты (в которых не затрагиваются решеточные операции). Так, для доказательства компактности пучка, индуцированного открытым семейством $l$-идеалов $0(M), M \in \operatorname{Max} S$, гельфандова $f$-полукольца используется лемма 3 пучковый вариант [11, теорема 4.3.1] теоремы Стоуна - Вейерштрасса о подалгебре банаховой алгебры.

Важной при изучении компактных пучков является информация о строении неприводимых и особенно максимальных $l$-идеалов $d r l$-полукольца $\Gamma(П)$ всех глобальных сечений. Предложение 3 описывает компактный пучок $(\Pi, X)$ функциональных полуколец в терминах непрерывных отображений неприводимого и максимального спектров $f$-полукольца сечений $\Gamma(\Pi)$ на базисное пространство $X$. Из данного предложения следует, что базисное пространство компактного пучка - это фактор-пространство как неприводимого, так и максимального спектров. Наконец, предложение 3 подсказывает, в каком случае и как для произвольного $f$-полукольца можно получить изоморфное представление в компактном пучке. В итоге имеем результат в виде теоремы 1. Приведены примеры, демонстрирующие применение теоремы 1.

В статье [4] авторами получены пучковые характеризации для некоторых функциональных полуколец, именно для риккартовых, бирегулярных, строго регулярных $f$-полуколец. В [4, теорема 2] было получено изоморфное представление гельфандова $f$-полукольца, однако, характеризация гельфандова $f$-полукольца в терминах сечений пучков осталась одной из нерешенных задач. Выводы о строении максимальных $l$-идеалов компактного пучка $f$-полуколец позволяют сформулировать заключительный результат статьи - теорему 2.

\section{1. Начальные понятия $d r l$-полуколец и пучковых представлений}

Напомним основные понятия и обозначения, используемые в статье.

Под полукольцом мы понимаем алгебру, отличающуюся от ассоциативного кольца, возможно, необратимостью аддитивной операции; не предполагается наличие единицы.

О п р е д е л е н и е $1[1]$. Алгебра $(S,+, \cdot, \vee, \wedge,-, 0)$ называется $d r l$-полукольцом, если выполняются условия:

(1) $(S,+, \cdot, 0)$ - полукольцо;

(2) $(S, \vee, \wedge)$ - решетка (с порядком $\leq)$; 
(3) сложение + дистрибутивно относительно $\vee$ и $\wedge$;

(4) $a-b-$ наименьший элемент $z$ такой, что $b+z \geq a$;

(5) $(a-b) \vee 0+b \leq a \vee b$ для любых $a, b \in S$;

(6) $a(b-c)=a b-a c$ и $(a-b) c=a c-b c$ для любых $a, b, c \in S$;

(7) $a b \geq 0$ для любых $a, b \geq 0$ из $S$.

Пусть $S-d r l$-полукольцо. Элемент $a \in S$, такой что $a \geq 0$, называется положительным. Множество всех положительных элементов из $S$ обозначается через $S^{+}$. Положительная часть элемента $a \in S-$ это элемент $a^{+}=a \vee 0$.

Cимметрическая разность на $d r l$-полукольце $S$ определяется как операция $a * b=(a-b) \vee$ $(b-a)$. Обозначим модуль элемента $a \in S$ через $|a|=a * 0=a \vee 0-a \wedge 0$.

Непустое подмножество $A d r l$-полукольца $S$ называется $l$-идеалом, если $A$ - идеал полукольца $(S,+, \cdot)$ и выпуклая подрешетка решетки $(S, \vee, \wedge)$ одновременно; последнее характеризуется условием: если $a \in A$ и $|s| \leq|a|$, то $s \in A$. Фактор- $d r l$-полукольцо по $l$-идеалу $A$ обозначается как $S / A$.

Элементы $a, b d r l$-полукольца называются ортогональными, если $|a| \wedge|b|=0$. Множество $A^{*}$ всех элементов $d r l$-полукольца $S$ ортогональных к каждому элементу подмножества $A \subseteq S$ является $l$-идеалом. Через $a^{*}$ обозначаем $\{a\}^{*}$ и $a^{* *}=\left(a^{*}\right)^{*}$.

Функциональным полукольцом (или, короче, f-полукольцом) называется $d r l$-полукольцо, являющееся подпрямым произведением линейно упорядоченных $d r l$-полуколец. Важным свойством $f$-полуколец является следующее:

$$
\text { если } a \wedge b=0 \text { и } c \geq 0 \text {, то } c a \wedge b=a c \wedge b=0 .
$$

В настоящей статье нас будут интересовать $d r l$-полукольца с единицей, поэтому определение пучка выглядит следующим образом.

О п р е д е л е н и е 2. Тройка $(P, \pi, X)$ называется пучком $d r l$-полуколеи, с единицей, если выполняются условия:

(1) $X$ и $P$ - топологические пространства, называемые соответственно базисным и накрывающим пространствами;

(2) $\pi: P \rightarrow X-$ локальный гомеоморфизм;

(3) для каждой точки $x \in X$ слой $P_{x}=\pi^{-1}(x)$ является $d r l$-полукольцом с единицей $1_{x}$, и $P=\dot{\cup} P_{x}, x \in X$

(4) $d r l$-полукольцевые операции непрерывны в $P$.

(5) отображение $X \rightarrow \Pi$, переводящую каждую точку $x \in X$ в единицу $1_{x}$ слоя $\Pi_{x}$, непрерывно.

Условие (5) определения пучка означает, что $d r l$-полукольцо Г(П) всех глобальных сечений пучка будет $d r l$-полукольцом с единицей. В общем случае (т. е. без требования выполнимости аксиомы (5)) наличие единицы в каждом слое пучка не гарантирует наличие единицы в алгебре глобальных сечений. Например, каждый слой пучка Даунса - Хофмана для бирегулярного кольца без единицы - это кольцо с единицей, но единицы в кольце всех глобальных сечений этого пучка нет [14, Theorem I.ii)].

Для построения пучков $d r l$-полуколец мы используем конструкцию для универсальных алгебр, идущую от Дэйви [15]. Именно семейство $\left\{A_{x}: x \in X\right\} l$-идеалов $d r l$-полукольца $S$, индексированных точками топологического пространства $X$, называется открытым, если множество $E_{a}=\left\{x \in X: a \in A_{x}\right\}$ открыто в $X$ для любого элемента $a \in S$. Для открытого семейства $l$-идеалов получаем пучок $(\Pi, X)$, где накрывающее пространство П является дизъюнктным объединением $d r l$-полуколец $S / A_{x}$ по всем точкам базисного пространства $X$.

Множество Irr $S$ всех неприводимых $l$-идеалов из $S$ образует топологическое пространство с открытыми множествами вида $D(A)=\{P \in \operatorname{Irr} S: P \nsupseteq A\}, A-l$-идеал из $S$, и называется неприводимым спектром $d r l$-полукольца $S$. K его важным подпространствам относятся максимальный $\operatorname{Max} S$ и минимальный $\operatorname{Min} S$ спектры - пространства всех максимальных $l$-идеа- 
лов и минимальных неприводимых $l$-идеалов. Открытые множества в этих подпространствах обозначаем через $D_{1}(A)=D(A) \cap \operatorname{Max} S$ и $d(A)=D(A) \cap \operatorname{Min} S$.

Для более подробной информации о $d r l$-полукольцах и их пучковых представлениях мы отсылаем к статьям [3-5].

Комментарий $\kappa$ статъе [4]. В статье [4] не было указано, что любое строго регулярное $f$-полукольцо по определению является риккартовым $f$-полукольцом. В указанной работе используется правильное определение строго регулярного $f$-полукольца. Авторы приносят извинения за допущенную неточность.

\section{2. Компактные пучки}

Пусть $(\Pi, X)$ - пучок $d r l$-полуколец с единицей, слои будем обозначать через $\Pi_{x}, x \in X$, нуль и единицу слоя $\Pi_{x}$ - через $0_{x}$ и $1_{x}$ и $\Gamma=\Gamma(\Pi, X)$ есть $d r l$-полукольцо всех глобальных сечений.

Подмножества $A$ и $B$ пространства $X$ называются $Г$-отделимыми, если найдется такое сечение $\varphi \in \Gamma$, что $\varphi(A)=0$ и $\varphi(B)=1$, т. е. для любых $x \in A$ и $y \in B$ выполняется $\varphi(x)=0_{x}$ и $\varphi(y)=1_{y}$.

О п р е д е л е н и е 3 . Пучок (П, $X)$ полуколец (или $d r l$-полуколец) назовем:

$\Gamma$-отделимым, если Г-отделимы любые две различные точки $x$ и $y$ из $X$;

$\Gamma$-регулярным, если Г-отделимы любые замкнутое множество $A \subseteq X$ и точка $x \in X \backslash A$;

Г-нормальным, если Г-отделимы любые два замкнутые непересекающиеся подмножества пространства $X$.

Непосредственно из определения следует, что Г-отделимость пучка влечет хаусдорфовость его базисного пространства $X$. А поскольку одноточечные множества $T_{1}$-пространства замкнуты, то для пучка над $T_{1}$-пространством $\Gamma$-нормальность влечет $\Gamma$-регулярность, которая влечет Г-отделимость.

Напомним, что пучок $(\Pi, X)$ называется факторным, если через каждую точку накрывающего пространства П проходит хотя бы одно глобальное сечение; другими словами, отображение $f_{x}: \Gamma(\Pi) \rightarrow \Pi_{x}$, сопоставляющее каждому глобальному сечению его значение в точке $x \in X$, является эпиморфизмом. В теории пучковых представлений алгебр наибольшее значение имеют именно факторные пучки: каждый пучок можно ограничить до факторного, при этом алгебра всех глобальных сечений останется такой же, как и в исходном пучке. Если $(\Pi, X)$ - факторный пучок $d r l$-полуколец, то ядро эпиморфизма $f_{x}$ есть $l$-идеал $J_{x}$ и произвольный слой П $x$ пучка изоморфен фактор- $d r l$-полукольцу $\Gamma(\Pi) / J_{x}$.

Лемма 1. Произвольный Г-отделимый пучок drl-полуколеи, над компактным базисным пространством является факторным.

Д о к а з а т е л ь с т в о. Пусть $p \in \Pi_{x}$ - произвольная точка накрывающего пространства. По определению пучка всегда есть локальное сечение $\varphi$, определенное на некотором открытом множестве $U \subseteq X$ и проходящее через точку $p$. Для любой точки $y \in X \backslash U$ найдется такое глобальное сечение $\sigma_{y}$, что $\sigma_{y}(x)=1_{x}$ и $\sigma_{y}(y)=0_{y}$. Нуль-множества $z\left(\sigma_{y}\right)=\{t \in X$ : $\left.\sigma_{y}(t)=0_{t}\right\}, y \in X \backslash U$, образуют открытое покрытие замкнутого множества $X \backslash U$, поэтому в силу компактности выберем его конечное подпокрытие. Пусть $z\left(\sigma_{1}\right) \cup \ldots \cup z\left(\sigma_{k}\right) \supseteq X \backslash U$ и для каждого $i$ выполняется $\sigma_{i}(x)=1_{x}$. Сечение $\sigma=\sigma_{1} \cdot \ldots \cdot \sigma_{k}$ совпадает с нулевым сечением на $U$ и $\sigma(x)=1_{x}$. Рассмотрим отображение

$$
\gamma=\left\{\begin{array}{l}
\sigma \varphi \text { на } U \\
0 \text { на } X \backslash U .
\end{array}\right.
$$

Оно является глобальным сечением. Действительно, $\gamma$ непрерывно на $U$, поскольку является произведением двух локальных сечений $\varphi$ и $\left.\sigma\right|_{U}$, и непрерывно на множестве $X \backslash U$, так как совпадает на нем с нулевым сечением. Наконец, заметим, что $\gamma(x)=p$. 
Лемма 2. Пусть (П, $X)$ - Г-отделимый пучок drl-полуколеи, над компактным пространством $X, U$ - замкнутое подмножество в $X$ и $x \in X \backslash U$. Тогда найдутся такие сечения $\alpha, \beta \in \Gamma(\Pi)$, чmo $0 \leq \alpha, \beta \leq 1, \alpha(x)=0_{x}, \beta(x)=1_{x},\left.\alpha\right|_{U}=1,\left.\beta\right|_{U}=0 u \alpha+\beta=1$.

Д о к а з а т е л ь с т в о. Для любой точки $y \in U$ в силу леммы 1 найдется такое глобальное сечение $\beta_{y}$, что $\beta_{y}(x)=1_{y}$ и $\beta_{y}(y)=0_{y}$. Нуль-множества $z\left(\beta_{y}\right), y \in U$, покрывают $U$, поэтому существует подпокрытие $z\left(\beta_{1}\right) \cup \ldots \cup z\left(\beta_{k}\right) \supseteq U$ для таких $\beta_{i}$, что $\beta_{i}(x)=1_{x}$ для всех $i=1, \ldots, k$. Сечение $\beta=\left(\beta_{1} \wedge \ldots \wedge \beta_{k} \wedge 1\right) \vee 0$ очевидно удовлетворяет условиям $0 \leq \beta \leq 1$, $\beta(x)=1_{x},\left.\beta\right|_{U}=0$. Тогда $\alpha=1-\beta$ является другим искомым сечением.

Напомним, что пучок (П, $X)$ называется мягким, если для любого замкнутого множества $N \subseteq X$ ограничение $\Gamma(\Pi) \rightarrow \Gamma\left(\left.\Pi\right|_{N}\right)$ есть эпиморфизм; другими словами, каждое сечение над замкнутым множеством продолжается до некоторого глобального сечения. Под носителем сечения $\sigma \in \Gamma(\Pi)$ понимается множество $\operatorname{supp} \sigma=\left\{x \in X: \sigma(x) \neq 0_{x}\right\}$. Носитель любого глобального сечения замкнут в $X$.

О п р е д е л е н и е 4. Пучок $(\Pi, X)$ называется Г-паракомпактным, если для каждого открытого покрытия $\left\{U_{i}: i \in I\right\}$ пространства $X$ существует подчиненное ему разбиение единицы - семейство глобальных сечений $\left\{\varphi_{j}: j \in J\right\}$, таких, что носитель каждого сечения $\varphi_{j}$ лежит в некотором множестве $U_{i}$, каждая точка из $X$ обладает окрестностью, пересекающейся только с конечным числом носителей $\operatorname{supp} \varphi_{i}$, и $\sum_{j \in J} \varphi_{j}=1-$ единичное сечение в $Г(П)$.

Данное определение применимо как для пучков колец или полуколец, так и для пучков $d r l$ полуколец с единицей. Оно корректно, поскольку второе условие обеспечивает суммируемость любого подсемейства из $\left\{\varphi_{j}: j \in J\right\}$.

Через $J_{x}$ обозначим множество всех глобальных сечений пучка $(\Pi, X) d r l$-полуколец, равных нулю в точке $x \in X$. Для произвольного пучка $(\Pi, X) d r l$-полуколец множество $J_{x}$ является $l$-идеалом $d r l$-полукольца $\Gamma(\Pi)$. Действительно, очевидно, что $J_{x}$ является полукольцевым идеалом. Пусть $|\beta| \leq|\alpha|$ и $\alpha \in J_{x}$. Тогда $|\beta(x)| \leq|\alpha(x)|=0_{x}$, откуда $\beta(x)=0_{x}$, т. е. $\beta \in J_{x}$.

Предложение 1. Пусть $(\Pi, X)$ - пучок drl-полуколеи, с единищей над компактом $X$. Тогда следующие условия равносильны:

(1) пучок П - Г-отделимый;

(2) пучок П - Г-регулярный;

(3) пучок П - Г-нормальный;

(4) П - мягкий пучок;

(5) пучок П - Г-паракомпактный;

(6) $J_{x}+J_{y}=\Gamma(\Pi)$ для любых различных $x, y \in X$.

Д о к а з а т е л ь с т в о. По [11, теорема 4.1.1] для произвольного пучка полуколец с единицей над компактом $X$ равносильны условия (1)-(4), (5) и (6), а условие (6) влечет (1). Пусть $(\Pi, X)-\Gamma$-отделимый пучок $d r l$-полуколец над компактом $X$, и $x \neq y \in X$. В силу хаусдорфовости $X$ найдется открытая окрестность $U$ точки $x$, не содержащая $y$. По лемме 2 найдутся такие сечения $\alpha, \beta \in \Gamma(\Pi)$, что $\alpha \in J_{x},\left.\beta\right|_{X \backslash U}=0$ и $\alpha+\beta=1$. Поскольку $y \in X \backslash U$, то $\beta \in J_{y}$. Следовательно, $J_{x}+J_{y}=\Gamma(\Pi)$, и доказана импликация $(1) \Rightarrow(6)$.

О п р е д е л е н и е 5 . Пучок $(\Pi, X) d r l$-полуколец с единицей над компактом $X$, удовлетворяющий равносильным условиям предложения 1 , назовем компактным.

Пример [11, с. 173] показывает различие компактных пучков полуколец и компактных пучков $d r l$-полуколец, а именно, для абстрактных полуколец Г-отделимость пучка - более слабое условие, чем условие (6) предложения 1.

П р и м е р ы. 1) Пучок (П, $X) d r l$-полуколец с единицей над нульмерным компактом (т. е. хаусдорфовым компактным пространством с базой открыто-замкнутых множеств) является компактным. Действительно, пусть $x, y$ - различные точки из $X$. Выберем произвольную открыто-замкнутую окрестность $U$ точки $x$, не содержащую точку $y$. Характеристическая 
функция $\varphi$, равная 1 на $U$ и 0 на $X \backslash U$, есть глобальное сечение пучка. Тогда для глобального сечения $\psi=1-\varphi$ выполняется $\varphi+\psi=1$, а поскольку $\varphi \in J_{y}$ и $\psi \in J_{x}$, то $J_{x}+J_{y}=\Gamma(\Pi)$.

2) $f$-Полукольцо $S$ называется риккартовым, если $a^{*}+a^{* *}=S$ для любого элемента $a \in S$ (см. [4]). Пусть $S$ - риккартово $f$-полукольцо с единицей. Тогда пучок $(L(S), \operatorname{Min} S)$ (см. п. 4 $[4])$ является компактным. Более того, пучок $(L(S), \operatorname{Min} S)$ компактен и для произвольного $f$-полукольца с единицей, это следует из [4, предложение 8].

3) Пучок $(\Psi(S), \operatorname{Max} \beta S)$ компактен для произвольного $d r l$-полукольца $S$ с единицей (см. [5, п. 3]).

Примеры 2) и 3) справедливы в силу того, что базисные пространства указанных пучков суть нульмерные компакты. Но нульмерность базисного пространства не является необходимым условием для компактности пучка. Чтобы продемонстрировать это, воспользуемся результатом, справедливым для пучков абстрактных полуколец.

Пусть $A$ - подполукольцо полукольца всех глобальных сечений пучка (П, $X)$ полуколец с единицей. Факторность подполукольца $A$ означает, что через каждую точку накрывающего пространства пучка проходит хотя бы одно глобальное сечение, лежащее в $A$. Для произвольной точки $x \in X$ введем обозначение: $O_{x}=\left\{s \in A: s(x)=0_{x}\right\}$. Следующий результат справедлив для пучков полуколец, не обязательно являющихся решеточно упорядоченными.

Лемма 3 [11, теорема 4.3.1]. Пусть $(\Pi, X)$ - пучок полуколеи, над компактным пространством $X, A$ - факторное подполукольцо полукольца $\Gamma(\Pi)$ и $O_{x}+O_{y}=A$ для любых различных $x, y \in X$. Тогда $(\Pi, X)$ - компактный пучок полуколеи, и $A=\Gamma(\Pi)$.

П р и м е р. $f$-Полукольцо $S$ называется гельфандовым, если для любой пары различных максимальных $l$-идеалов $M$ и $N$ из $S$ найдутся такие элементы $a \in M \backslash N$ и $b \in N \backslash M$, что $|a| \wedge|b|=0$. Рассмотрим гельфандово $f$-полукольцо $S$ с единицей. Пусть $(L(S), \operatorname{Max} S)$ - пучок $f$-полуколец, индуцированный открытой системой $l$-идеалов

$$
0(M)=\left\{s \in S: s^{*} \nsubseteq M\right\}, \quad M \in \operatorname{Max} S .
$$

Поскольку пересечение всех $l$-идеалов $0(M)$ нулевое [4, предложение 7], то представление $S$ сечениями этого пучка точное (инъективное), и $S \cong \hat{S}$. В силу этого для любого $M \in \operatorname{Max} S$ $l$-идеалы $O_{M}$ и $0(M)$ канонически изоморфны. По предложению $6[4] 0(M)+0(N)=S$ для любых различных максимальных $l$-идеалов $M, N$, поэтому $O_{M}+O_{N}=\hat{S}$. В силу строения пучка подполукольцо $\hat{S}$ полукольца всех глобальных сечений является факторным, а $\operatorname{Max} S-$ компактное пространство [4, предложение 7]. По лемме 3 получаем, что $(L(S), \operatorname{Max} S)$ - компактный пучок, но его базисное пространство не обязательно является нульмерным компактом.

Заметим, что кроме компактности пучка лемма 3 позволяет обосновать изоморфность представления гельфандова $f$-полукольца с единицей сечениями пучка $(L(S), \operatorname{Max} S)$. Это достигается благодаря наличию аддитивной характеризации гельфандова $f$-полукольца $[4$, предложение 6].

\section{3. Компактные пучки $f$-полуколец}

Дальнейшие наши исследования будут касаться пучков функциональных полуколец. Напомним, что собственный $l$-идеал $P d r l$-полукольца $S$ называется неприводимым, если $A \cap B \subseteq P$ влечет $A \subseteq P$ или $B \subseteq P$ для любых $l$-идеалов $A, B$ из $S$. В $f$-полукольце неприводимость $l$-идеала $P$ характеризуется через элементы: для любых $a, b \in S^{+} a \wedge b \in P$ влечет $a \in P$ или $b \in P[4$, предложение 3]. Произвольный максимальный $l$-идеал $d r l$-полукольца является неприводимым.

Выше нами было установлено, что для пучка $(\Pi, X)$ множество $J_{x}=\left\{\sigma \in \Gamma(\Pi): \sigma(x)=0_{x}\right\}$ есть $l$-идеал. 
Лемма 4. Пусть (П, $X)$ - компактный пучок $f$-полуколеи, и $P$ - неприводимый l-идеал f-полукольца Г(П). Тогда существует единственная точка $x \in X$, такал, что $J_{x} \subseteq P$.

Д о к а з а т е л ь с т в о. Предположим, что для каждой точки $x \in X$ выполняется условие $J_{x} \nsubseteq \subseteq P$ и, следовательно, для любой точки $x$ найдется сечение $\sigma_{x} \in J_{x} \backslash P$. Заметим, что $\sigma_{x} \vee 0 \in J_{x} \backslash P$, поэтому будем считать, что $\sigma_{x} \geq 0$. Нуль-множества сечений $\sigma_{x}, x \in X$, покрывают $X$, исходя из чего, воспользовавшись компактностью $X$, выберем конечное подпокрытие. Тогда $z\left(\sigma_{1}\right) \cup \ldots \cup z\left(\sigma_{k}\right)=X$, и для любого $x \in X$ есть такой индекс $i$, что $\sigma_{i} \in J_{x} \backslash P$. В силу неприводимости $P$ получаем $\sigma=\sigma_{1} \wedge \ldots \wedge \sigma_{k} \notin P$. Но, с другой стороны, $z(\sigma)=X$, поэтому $\sigma=0$, противоречие. Следовательно, для любого неприводимого $l$-идеала $P$ из $\Gamma(П)$ найдется такая точка $x$, что $J_{x} \subseteq P$. Если $J_{x}, J_{y} \subseteq P$ для различных $x, y \in X$, то $\Gamma(\Pi)=J_{x}+J_{y} \subseteq P$; противоречие.

Лемма 5. Пусть $(\Pi, X)-$ компактный пучок $f$-полуколеи, $f_{x}: \Gamma(\Pi) \rightarrow \Pi_{x}-$ естественный эпиморфизм, $P$ - неприводимый l-идеал f-полукольиа $\Gamma(\Pi)$ u $J_{x} \subseteq P$. Тогда $f_{x}(P) \neq \Pi_{x}$.

Д о к а з а т е л ь с т в о. Допустим, что $f_{x}(P)=\Pi_{x}$. Тогда существует сечение $\rho \in P$ такое, что $\rho(x)=1_{x}$ и по свойствам пучков $\rho$ совпадает с единичным сечением на некоторой открытой окрестности $U$ точки $x$. По лемме 2 найдется сечение $\gamma$ такое, что $0 \leq \gamma \leq 1$, $\gamma(x)=0_{x},\left.\gamma\right|_{U}=1$. Если $y \in U$, то

$$
\rho(y)+\gamma(y)=1_{y}+1_{y} \gamma(y)=1_{y}+\rho(y) \gamma(y) .
$$

Если $y \notin U$, то

$$
\rho(y)+\gamma(y)=\rho(y) 1_{y}+1_{y}=1_{y}+\rho(y) \gamma(y) .
$$

Доказали справедливость равенства $\rho+\gamma=1+\rho \gamma$. По лемме 2 найдутся такие сечения $\alpha, \beta$, что $\alpha \in J_{x} \subseteq P,\left.\beta\right|_{X \backslash z(\gamma)}=0$ и $\alpha+\beta=1$. Сечение $\beta$ не лежит в $P$, иначе $1 \in P$. Заметим, что $\gamma \beta=0$, поскольку объединение нуль-множеств этих сечений есть все пространство $X$. Получаем $\rho \beta=(\rho+\gamma) \beta=(1+\rho \gamma) \beta=\beta$; противоречие, так как $\rho \beta \in P$, а $\beta \notin P$.

Пусть $(\Pi, X)$ - пучок $f$-полуколец, $x \in X$ и $M$ - максимальный $l$-идеал слоя $\Pi_{x}$. Рассмотрим множество

$$
M_{x}=\{\varphi \in \Gamma(\Pi): \varphi(x) \in M\} .
$$

Предложение 2. Максимальными l-идеалами f-полукольца Г(П) глобальных сечений компактного пучка $f$-полуколеч, $(\Pi, X)$ являются в точности l-идеаль $M_{x}$, различные для различных $\operatorname{nap}(M, x)$.

Д о к а з а т е л ь с т в о. То, что $M_{x}$ есть $l$-идеал проверяется непосредственно. Допустим, $M_{x}$ лежит в некотором собственном $l$-идеале $N$ из $\Gamma(\Pi)$. Тогда $J_{x} \subseteq N$. По лемме $5 f_{x}(N) \neq \Pi_{x}$, и получаем $M=f_{x}\left(M_{x}\right) \subseteq f_{x}(N)$, т. е. $M=f_{x}(N)$. Отсюда $N \subseteq M_{x}$, и $M_{x}-$ максимальный $l$-идеал в $\Gamma$.

Обратно, пусть $N$ - максимальный $l$-идеал в $Г(П)$. Тогда согласно лемме $4 J_{x} \subseteq N$ для однозначно определенной точки $x \in X$. Поскольку по лемме $5 l$-идеал $f_{x}(N)$ является собственным в слое $\Pi_{x}$, то $f_{x}(N)$ лежит в некотором максимальном $l$-идеале $M$ слоя $\Pi_{x}$. Тогда $N \subseteq M_{x}$, откуда получаем $N=M_{x}$ в силу максимальности $N$.

Напомним, что элемент $d r l$-полукольца $S$ называется формальной единицей, если он не лежит ни в одном собственном $l$-идеале из $S$.

Следствие 1. Пусть (П, $X)-$ компактный пучок $f$-полуколец, и $\sigma \in \Gamma(\Pi)$. Тогда равносильны следующие условия:

(1) $\sigma-$ формальная единица Г(П);

(2) $\sigma(x)-$ формальная единича каждого слоя $\Pi_{x}$. 
Пусть $P$ - неприводимый $l$-идеал $f$-полукольца $\Gamma=\Gamma(\Pi)$ глобальных сечений компактного пучка $(\Pi, X)$. По лемме $4 J_{x} \subseteq P$ для однозначно определенной точки $x \in X$. Поэтому

$$
h: \operatorname{Irr} \Gamma \rightarrow X, \text { где } h(P)=x \Leftrightarrow J_{x} \subseteq P,
$$

является отображением топологических пространств. У каждой точки $x \in X$ есть прообраз $M_{x}, M \in \operatorname{Max} \Pi_{x}$, отсюда $h-$ сюръективное отображение. Ограничение отображения $h$ на Мах Г задает отображение

$$
g: \operatorname{Max} \Gamma \rightarrow X
$$

при котором $M_{x}$ имеет образ $x \in X$.

Лемма 6. Пусть $(\Pi, X)$ компактный пучок $f$-полуколец, с единицей. Тогда отображение $h$ непреръвно.

Д о к а з а т е л ь с т в о. Пусть $U$ - открытое в $X$ множество и $P \in h^{-1}(U)$. Точка $x=h(P)$ лежит в $U$, и в силу Г-регулярности пучка найдется сечение $\varphi \in \Gamma(\Pi)$ такое, что $\varphi(x)=1_{x}$ и $\left.\varphi\right|_{X \backslash U}=0$. Поскольку $J_{x} \subseteq P$, то по лемме 5 выполняется $1_{x} \notin f_{x}(P)$, где $f_{x}: \Gamma(\Pi) \rightarrow \Pi_{x}-$ естественный эпиморфизм. Поэтому $\varphi \notin P$. Пусть $B=\cap\left\{J_{y}: y \in X \backslash U\right\}$ есть $l$-идеал $f$-полукольца $\Gamma$. Поскольку $\varphi \in B$, то $B \nsubseteq P$ и $P \in D(B)$. Показали включение $h^{-1}(U) \subseteq D(B)$. Обратно, пусть $P \in D(B)$. Тогда существует глобальное сечение $\varphi \in B \backslash P$. Для точки $x=h(P)$ имеем $\varphi \notin J_{x}$, так как в противном случае $\varphi \in P$. Следовательно, $h(P)=$ $x \notin X \backslash U$, т. е. $h(P) \in U$, и $P \in h^{-1}(U)$. Получили $D(B) \subseteq h^{-1}(U)$, откуда $D(B)=h^{-1}(U)$, и $h$ - непрерывное отображение.

Предложение 3. Пусть $(П, X)$ - пучок $f$-полуколеи, с единицей. Равносильны утверждения:

(1) $(\Pi, X)$ - компактный пучок $f$-полуколеи;

(2) для любого неприводимого l-идеала $P$ из $\Gamma(\Pi)$ найдется единственная точка $x \in X$ такая, что $J_{x} \subseteq P$ и соответствующее отображение $h: \operatorname{Irr} \Gamma(\Pi) \rightarrow X, h(P)=x$, непрерывно и сюргективно;

(3) для любого максимального $l$-идеала $M$ из $Г(П)$ найдется единственная точка $x \in X$ такал, что $J_{x} \subseteq M$ и соответствующее отображение $g: \operatorname{Max} \Gamma(\Pi) \rightarrow X, g(M)=x$, непрерывно и сюргективно.

Д о к а з а т е л ь с т в о. Импликация $(1) \Rightarrow(2)$ следует из лемм 5 и 6 , а сюръективность отображения $h$ была обоснована выше.

Рассмотрим $(2) \Rightarrow(1)$. Пусть $x, y$ - различные точки пространства $X$. Тогда $l$-идеал $J_{x}+J_{y}$ не лежит ни в каком неприводимом, в частности ни в каком максимальном, $l$-идеале, поэтому $J_{x}+J_{y}=\Gamma(\Pi)$. Пусть $\varphi+\psi=1$ для подходящих глобальных сечений из $J_{x}$ и $J_{y}$. Нульмножества $z(\varphi)$ и $z(\psi)$ открыты, не пересекаются и содержат точки $x$ и $y$ соответственно. Показали, что $X$ хаусдорфово. Пространство $X$ компактно как образ компактного пространства Irr $\Gamma$ при непрерывном отображении $h$.

Импликация (1) $\Leftrightarrow(3)$ доказывается аналогично.

Предложение 3 дает идею получения компактного представления произвольного $f$-полукольца.

Теорема 1. Пусть $S-f$-полукольио с единищей и выполняется хотя бы одно из условий:

(1) $h: \operatorname{Irr} S \rightarrow X-$ непрерывное отображсение на компакт $X$;

(2) $g: \operatorname{Max} S \rightarrow X-$ непрерывное отображсение на компакт $X$.

Тогда в каждом случае существует такой компактный пучок (П, $X)$, что $S \cong \Gamma(\Pi)$. 
Д о к а з а т е л ь с т в о теоремы разобьем на несколько вспомогательных утверждений. Для $x \in X$ определим

$$
\begin{gathered}
J_{x, h}=\left\{a \in S:\left(\exists U_{a}-\text { открытая окрестность } x\right)\right. \\
\left.\left(\forall P \in \operatorname{Irr} S\left(h(P) \in U_{a}\right)\right)(\exists b \notin P(|a| \wedge b=0))\right\} .
\end{gathered}
$$

Отталкиваясь от отображения $g: \operatorname{Max} S \rightarrow X$, аналогичным образом задаем множество $J_{x, g}$.

Назовем множество $U_{a}$, указанное в определениях, подходящим для элемента $а$ (из $J_{x, h}$ или $\left.J_{x, g}\right)$. Ясно, что любая открытая окрестность точки $x$, лежащая в произвольном подходящем для $a$ множестве, также является подходящим для $a$ множеством.

Лемма 7. Eсли $h(P) \neq h(Q)$, то найдутся такие $a \in S \backslash P$ u $b \in S \backslash Q$, что $a \wedge b=0$.

Д о к а з а т е л ь с т в о. Пусть $x=h(P) \neq h(Q)=y$. Найдутся непересекающиеся открытые окрестности $U$ и $V$ точек $x$ и $y$ соответственно. Тогда $h^{-1}(U)$ и $h^{-1}(V)$ - непересекающиеся открытые окрестности в $\operatorname{Irr} S$ точек $P$ и $Q$. Отсюда следует, что $l$-идеалы $P$ и $Q$ являются несравнимыми (т. е. не выполняется ни $P \subseteq Q$, ни $Q \subseteq P$ ). Как доказано в лемме 11 из [4] найдутся такие $a \in S \backslash P$ и $b \in S \backslash Q$, что $a \wedge b=0$.

Следствие 2. Если $P, Q$ - неприводимые l-идеаль и выполняется $P \subseteq Q$, mо $h(P)=$ $h(Q)$.

Лемма 8. $J_{x, g}$ и $J_{x, h}$ являются l-идеалами.

Д о к а з а т е л ь с т в о. Пусть $a_{1}, a_{2} \in J_{x, h}$ и $U_{1}, U_{2}$ - подходящие множества для элементов $a_{1}$ и $a_{2}$ соответственно. Тогда для любого $P \in \operatorname{Irr} S$ с условием $h(P) \in U_{1} \cap U_{2}$ найдутся такие $b_{1}, b_{2} \notin P$, что $\left|a_{1}\right| \wedge b_{1}=\left|a_{2}\right| \wedge b_{2}=0$. В силу неприводимости $l$-идеала $P$ выполняется $b=b_{1} \wedge b_{2} \notin P$ и, кроме того, $\left|a_{1}\right| \wedge b=\left|a_{2}\right| \wedge b=0$. Тогда

$$
0=\left(\left|a_{1}\right| \wedge b\right)+\left(\left|a_{2}\right| \wedge b\right)=\left(\left|a_{1}\right|+\left|a_{2}\right|\right) \wedge b \geq\left(\left|a_{1}+a_{2}\right|\right) \wedge b \geq 0
$$

это равенство является известным результатом, справедливым в любом решеточно упорядоченном моноиде [16, гл. 14, 4 , теорема 3], а доказательство неравенства можно найти в [17, предложение 3]. Таким образом, $a_{1}+a_{2} \in J_{x, h}$. Пусть $s \in S$. Из $\left|a_{1}\right| \wedge b_{1}=0$ в $f$-полукольце получаем $\left|a_{1}\right||s| \wedge b_{1}=0$ [5, предложение 4], а из [1, Proposition 1.1] $-0=\left|a_{1}\right||s| \wedge b_{1} \geq\left|a_{1} s\right| \wedge b_{1} \geq 0$. Следовательно, $\left|a_{1} s\right| \wedge b_{1}=0$, и $J_{x, h}-$ полукольцевой идеал. Наконец, если $|c| \leq\left|a_{1}\right|$, то $0 \leq|c| \wedge b_{1} \leq\left|a_{1}\right| \wedge b_{1}=0$, откуда имеем $c \in J_{x, h}$.

Доказательство для $J_{x, g}$ аналогичное.

В последующих леммах, как и в лемме 8 , нет принципиального различия в утверждениях и доказательствах об $l$-идеалах $J_{x, h}$ или $J_{x, g}$. Поэтому ниже обозначим через $J_{x} l$-идеал $J_{x, h}$. Доказательства утверждений о $J_{x, g}$ осуществляются заменой $h$ на $g$, неприводимых $l$-идеалов на максимальные, $\operatorname{Irr} S$ на $\operatorname{Max} S$.

Лемма 9. Справедливы следующие утверждения:

(1) $\left\{J_{x}: x \in X\right\}-$ открытая система l-идеалов;

(2) $\cap\left\{J_{x}: x \in X\right\}=0$.

Д о к а з а т е л ь с т в о. (1) Для произвольного элемента $a \in S$ докажем, что множество $U=\left\{x \in X: a \in J_{x}\right\}$ открыто в $X$. Пусть $y \in U$. Поскольку $a \in J_{y}$, то найдется открытая окрестность $V$ точки $y$, такая что для любого неприводимого $l$-идеала $P$ со свойством $h(P) \in V$ найдется элемент $b \notin P$, удовлетворяющий $|a| \wedge b=0$. Для произвольной точки $t \in V$ та же окрестность $V$ будет подходящей для элемента $a$, поэтому $V \subseteq U$. Поскольку каждая точка из $U$ имеет открытую окрестность, лежащую в $U$, то $U$ - открытое множество. 
(2) Если $a \in \cap\left\{J_{x}: x \in X\right\}$, то $l$-идеал $a^{*}$ не лежит ни в одном неприводимом $l$-идеале, поэтому $a^{*}=S$. Тогда $a \in a^{*}$, следовательно, $|a| \wedge|a|=0$ и $a=0$.

Таким образом, получаем два типа пучков $f$-полуколец

$$
\left(\dot{\cup} S / J_{x, h}, X\right) \text { и }\left(\dot{\cup} S / J_{x, g}, X\right) \text {. }
$$

Лемма 10. $E с л и ~ h(P)=x$, mo $J_{x} \subseteq P$.

Д о к а з а т е л ь с т в о. Предположим, что $h(P)=x$, но $P \nsupseteq J_{x}$. Тогда для элемента $a \in J_{x} \backslash P$ рассмотрим подходящую окрестность $U_{a}$, содержащую $x$. Поскольку $P \in h^{-1}\left(U_{a}\right)$, то выполняется $|a| \wedge b=0$ для некоторого элемента $b \notin P$; получили противоречие с неприводимостью $P$.

Лемма 11. Если $x \neq y$, mo $J_{x}+J_{y}=S$.

Д ок а з а т е л ь с т в о. Пусть $x \neq y$ и $h(Q)=y$ для некоторого $Q \in \operatorname{Irr} S$. По лемме $10 J_{y} \subseteq Q$. Покажем, что $J_{x} \nsubseteq Q$. Зафиксируем $W$ и $U-$ непересекающиеся открытые окрестности точек $x$ и $y$. Можно считать $U$ подходящим множеством для некоторого элемента из $J_{x}$. Множество $V=h^{-1}(X \backslash U)$ замкнуто в $\operatorname{Irr} S$ как прообраз замкнутого множества при непрерывном отображении. Пусть $P$ - такой произвольный неприводимый $l$ идеал, что $h(P)=x$. По лемме 7 для каждого $P^{\prime} \in V$ найдутся такие $a^{\prime} \notin P^{\prime}, b^{\prime} \notin Q$, что $a^{\prime} \wedge b^{\prime}=0$. Открытые множества $D\left(a^{\prime}\right)$ покрывают $V$, поэтому найдется конечное подпокрытие $D\left(a_{1}\right) \cup \ldots \cup D\left(a_{k}\right) \supseteq V$ и элементы $b_{1}, \ldots, b_{k}$, соответствующие элементам $a_{i}, i=1, \ldots, k$. Поскольку конечно порожденный $l$-идеал $d r l$-полукольца является главным [5, лемма 1], то $D\left(a_{1}\right) \cup \ldots \cup D\left(a_{k}\right)=D\left(\left(a_{1}\right)+\ldots+\left(a_{k}\right)\right)=D(a)$ для некоторого $a \in S$. Получаем, $a \notin P^{\prime}$ для любого $P^{\prime} \in V$, а $b=b_{1} \wedge \ldots \wedge b_{k} \notin Q$. По [1, prop. 1.4]

$$
|a| \leq \sum_{i=1}^{k}\left(m_{i} a_{i}+s_{i} a_{i}+a_{i} t_{i}+u_{i} a_{i} v_{i}\right)
$$

для некоторых целых неотрицательных $m_{i}$ и $s_{i}, t_{i}, u, v_{i} \in S^{+}$. Каждое слагаемое, а значит и их сумма, ортогональны элементу $b$ (рассуждения как и в лемме 8), поэтому $|a| \wedge|b|=0$. Для любого $P^{\prime} \in h^{-1}(W)$ имеем $a \notin P^{\prime}$, поэтому $W$ является подходящим множеством для элемента $b$. Поскольку $x \in W$, то $b \in J_{x}$. Но $b \notin Q$, значит, $J_{x} \nsubseteq \subseteq Q$.

Продолжим доказательство теоремы 1.

(1) По лемме 9 представление $S \rightarrow \hat{S}$ в пучке $(\Pi, X)=\left(\dot{\cup} S / J_{x, h}, X\right)$ является точным, и $f$-полукольцо $\hat{S}$ в силу строения пучка факторное. l-Идеалы $O_{x}$ (см. лемму 3 ) совпадают с $J_{x}$. По лемме 11 получаем, что выполнены все условия леммы 3 , поэтому $(\Pi, X)$ - компактный пучок $f$-полуколец и $S \cong \hat{S}=\Gamma(\Pi)$.

Пункт (2) доказывается так же, как и п. (1), заменой неприводимых $l$-идеалов на максимальные.

Теорема доказана.

Покажем, как используется теорема 1 для получения некоторых компактных представлений.

П р и м е р ы. 1) Пусть $S$ - гельфандово $f$-полукольцо. Тождественное отображение $g$ : $\operatorname{Max} S \rightarrow \operatorname{Max} S$ для любого $M \in \operatorname{Max} S$ задает $l$-идеал $J_{M, g}$. Убедимся, что $J_{M, g}=0(M)$. Действительно, очевидны импликации

$$
a \in J_{M, g} \Rightarrow a^{*} \nsubseteq M \Leftrightarrow a \in 0(M) .
$$

Если $a^{*} \nsubseteq M$, то $|a| \wedge b=0$ для некоторого $b \notin M$. Тогда $D_{1}(b)$ - подходящее множество для элемента $a$ и $M \in D_{1}(b)$. Тем самым справедлива импликация $a^{*} \nsubseteq M \Rightarrow a \in J_{M, g}$. Таким 
образом, получаем компактный пучок $(\dot{\cup} S / 0(M), \operatorname{Max} S)$ и $S$ изоморфно $f$-полукольцу всех его глобальных сечений. Прямое доказательство этого результата было приведено в [4, теорема 2].

2) Пусть $S$ - риккартово $f$-полукольцо с единицей. По [4, предложение 12$]$ имеется ретракция $h: \operatorname{Irr} S \rightarrow \operatorname{Min} S$ - непрерывное отображение, тождественное на $\operatorname{Min} S$. В пространстве $\operatorname{Min} S$ минимальных неприводимых $l$-идеалов открытые множества имеют вид $d(A)=$ $D(A) \cap \operatorname{Min} S$. Также отметим, что если в риккартовом $f$-полукольце для $M \in \operatorname{Min} S, P \in \operatorname{Irr} S$ выполняется $M \subseteq P$, то $M=0(M)=0(P)$ (по лемме 13 и предложению 11 [4]). Пусть $M \in \operatorname{Min} S$ и $a \in M$. Рассмотрим $d\left(a^{*}\right)$ - открытое множество в $\operatorname{Min} S$, содержащее $M$. Для $P \in \operatorname{Irr} S$ такого, что $h(P)=N \in d\left(a^{*}\right)$, имеем $0(P)=0(N)=N$. Поскольку $a^{*} \nsubseteq N$, то $a \in N$ [4, лемма 12], откуда $a \in 0(P)$. Следовательно, $a^{*} \nsubseteq \mathbb{P}$, и получаем $a \in J_{M, h}$. Включение $J_{M, h} \subseteq M$ очевидно, поэтому доказано $J_{M, h}=M$. Итак, получили пучок $(\dot{\cup} S / M, \operatorname{Min} S)$ $f$-полуколец с единицей и компактное представление риккартова $f$-полукольца $S$ с единицей сечениями этого пучка (см. также [4, теорема 3$])$.

3) Пусть $S$ - произвольное $d r l$-полукольцо. $l$-Идеал $A$ из $S$ называется дополняемым, если для некоторого $l$-идеала $B$ выполняется $A+B=S$ и $A \cap B=0$; в этом случае $B$ называется дополнением $\kappa A$, определяется однозначно и обозначается через $A^{\perp}$. Множество $\beta S$ всех дополняемых $l$-идеалов из $S$ является булевой решеткой. Через Мах $\beta S$ обозначаем пространство максимальных идеалов из $\beta S$ со стоуновской топологией; базис открыто-замкнутых множеств образуют множества вида

$$
D_{\beta}(A)=\{M \in \operatorname{Max} \beta S: M \nsupseteq A\}, \quad A \in \beta S .
$$

Пусть сейчас $S$ - произвольное $f$-полукольцо с единицей. Для максимального $l$-идеала $M$ из $S$ рассмотрим множество $M_{\beta}=\{A \in \beta S: A \subseteq M\}$. Стандартно проверяется, что $M_{\beta}$ является максимальным идеалом решетки $\beta S$. Получаем отображение

$$
g: \operatorname{Max} S \rightarrow \operatorname{Max} \beta S, \quad g(M)=M_{\beta} .
$$

Покажем, что $g^{-1}\left(D_{\beta}(A)\right)=D_{1}(A)$ для любого дополняемого $l$-идеала $A$. Действительно, справедлива следующая цепочка равносильных утверждений:

$$
M \in g^{-1}\left(D_{\beta}(A)\right) \Leftrightarrow M_{\beta} \in D_{\beta}(A) \Leftrightarrow A \notin M_{\beta} \Leftrightarrow A \nsubseteq M \Leftrightarrow M \in D_{1}(A) .
$$

Таким образом, $g$ - непрерывное отображение.

Если $T \in \operatorname{Max} \beta S$, то сумма $C$ всех дополняемых $l$-идеалов из $T$ будет собственным (в общем, не обязательно дополняемым) $l$-идеалом $f$-полукольца $S$. Тогда $C$ лежит в некотором максимальном $l$-идеале $M$ из $S$. Получаем $g(M)=M_{\beta}=T$, и $g$ - сюръективное отображение.

Выясним сейчас строение $l$-идеала $J_{M_{\beta}, g}$, который для краткости обозначим через $J_{M}$. Рассмотрим множество $0_{M}=\cup\left\{A \in \beta S: A \in M_{\beta}\right\}$, являющееся $l$-идеалом в $S$ (см. [5, лемма 5]). Покажем, что $0_{M}=J_{M}$. Пусть $a \in 0_{M}$, тогда $a$ лежит в некотором дополняемом $l$-идеале $A \in M_{\beta}$. Из $A+A^{\perp}=S$ получаем $u+v=1$ для некоторых $u \in A$ и $v \in A^{\perp}$. Поскольку $|a| \wedge|v| \in A \cap A^{\perp}=0$, то $v \in a^{*}$. Рассмотрим $D_{\beta}(v)$ - открыто-замкнутую окрестность точки $M_{\beta} \in \operatorname{Max} \beta S$. Для произвольного максимального $l$-идеала $N \in D_{1}(v)=g^{-1}\left(D_{\beta}(v)\right)$ получаем $(v) \nsubseteq N$, поэтому $a^{*} \nsubseteq N$, откуда $a \in J_{M}$. Обратно, пусть $a \in J_{M}$. Возьмем для некоторого дополняемого $l$-идеала $(v)$ окрестность $D_{\beta}(v)$ точки $M_{\beta}$, являющуюся подходящим множеством для элемента $a$. Тогда $a^{*}$ не лежит ни в каком $N \in g^{-1}\left(D_{\beta}(v)\right)=D_{1}(v)$. Для $l$-идеала $A=a^{*} \cap(v)$ имеем $D_{1}(A)=D_{1}(v)$, откуда очевидно следует $D(A)=D(v)$. Согласно лемме 4 из [4] получаем $A=(v)$, поэтому $(v) \subseteq a^{*}$. Отсюда $a \in(v)^{\perp}$, и поскольку $(v)^{\perp} \in M_{\beta}$, то $a \in 0_{M}$.

Показали, что для $f$-полукольца с единицей $S$ отображение $g: \operatorname{Max} S \rightarrow \operatorname{Max} \beta S$ задает компактный пучок (ن $\left.S / 0_{M}, \operatorname{Max} \beta S\right)$ и изоморфное представление $S$ его сечениями. Рассмотренный пример является частным случаем компактного пучка и представления, полученными для произвольного $d r l$-полукольца в [5, §3 и теорема 2]. 
Заключительный результат статьи посвящен пучковой характеризации гельфандова $f$-полукольца.

Справедливо следующее утверждение.

Теорема 2. Для того чтобы произвольное f-полукольцо с единичей было гельфандовым, необходимо и достаточно, чтобы оно было изоморфно f-полукольиу всех глобальных сечений компактного пучка f-полуколеи, с единицей, все слои которого имеют в точности по одному максимальному l-идеалу.

Д о к а з а т е л ь с т в о. Необходимость установлена в теореме 2 [4].

Пусть $(\Pi, X)$ - компактный пучок, каждый слой которого является $f$-полукольцом с единицей и единственным максимальным $l$-идеалом. Пусть $M_{x}, M_{y}$ - различные максимальные $l$-идеалы $f$-полукольца $\Gamma(П)$. Поскольку каждый слой пучка имеет единственный максимальный $l$-идеал, то по предложению 2 точки $x$ и $y$ различны. В силу компактности пучка $\sigma+\gamma=1$ для некоторых $\sigma \in J_{x}$ и $\gamma \in J_{y}$. Носитель $\operatorname{supp} \sigma$ замкнут и не содержит точку $x$. По лемме 2 найдется сечение $\varphi \geq 0$, равное нулю на $\operatorname{supp} \sigma$ и единице в точке $x$. Тогда по лемме $5 \varphi \notin M_{x}$. Далее, $\sigma^{+}(y)=\sigma(y)=1_{y}$, поэтому $\sigma^{+} \notin M_{y}$. Показали, что $\varphi \in M_{y} \backslash M_{x}$ и $\sigma^{+} \in M_{x} \backslash M_{y}$. Наконец, глобальные сечения $\varphi$ и $\sigma^{+}$положительны, их нуль-множества покрывают $X$, поэтому $\varphi \wedge \sigma^{+}=0$. Следовательно, $Г(\Pi)-$ гельфандово $f$-полукольцо.

Теорема доказана.

\section{Заключение}

Остались следующие открытые вопросы.

1) Как определить компактность пучка drl-полуколеи, без единицы? Одним из вариантов определения - отделимость точек накрывающего пространства, являющихся в своих слоях нулем и формальной единицей.

2) Справедлива ли теорема 1 в классе drl-полуколец? Возможно, необходимо рассматривать отображения $f$ и $g$, удовлетворяющие дополнительному условию, сформулированному в лемме 7 .

\section{СПИСОК ЛИТЕРАТУРЫ}

1. Rao P.R. Lattice ordered semirings // Math. Sem. Notes, Kobe Univ. 1981. Vol. 9. P. 119-149.

2. Swamy K.L.N. Dually residuated lattice ordered semigroups // Math. Ann. 1965. Vol. 159. P. 105-114. doi: $10.1007 /$ BF01360284

3. Чермных О.В. O drl-полугруппах и drl-полукольцах // Чебышевский сб. 2016. Т. 14, № 4. С. $167-$ 179. doi: 10.22405/2226-8383-2016-17-4-167-179.

4. Чермных В.В., Чермных О.В. Функциональные представления решеточно упорядоченных полуколец // Сиб. электрон. мат. известия. 2017. Т. 14. С. 946-971. doi: 10.17377/semi.2017.14.080 .

5. Чермных О.В. Функциональные представления решеточно упорядоченных полуколец. II // Сиб. электрон. мат. известия. 2018. Т. 15. С. 677-684. doi: 10.17377/semi.2018.15.053.

6. Pierce R.S. Modules over commutative regular rings // Mem. Amer. Math. Soc. 1967. Vol. 70. P. 1-112. doi: $10.1090 / \mathrm{memo} / 0070$.

7. Koh K. On a representation of strongly harmonic ring by sheaves // Pacif. J. Math. 1972. Vol. 41, no 2. P. 459-468. doi: 10.2140/pjm.1972.41.459.

8. Mulvey C.J. A generalization of Gelfand duality // J. Algebra. 1979. Vol. 56, no 2. P. 499-505. doi: 10.1016/0021-8693(79)90352-1.

9. Filipoiu A. Compact sheaves of lattices and normal lattices // Math. Jap. 1991. Vol. 36, no 2. P. 381386.

10. Чермных В.В. Представление положительных полуколец сечениями // Успехи мат. наук. 1992. T. 47, № 5. C. 180-182. doi: 10.1070/RM1992v047n05ABEH000948.

11. Чермных В.В. Функциональные представления полуколец // Фундам. и прикл. математика. 2012. T. 17, № 3. C. 111-227. doi: 10.1007/s10958-012-1062-2 . 
12. Mulvey C.J. Compact ringed spaces // J. Algebra. 1978. Vol. 52, no 2. P. 411-436. doi: 10.1016/00218693(78)90248-X.

13. Simmons H. Compact representations - the lattice theory of compact ringed spaces // J. Algebra. 1989. Vol. 126. P. 493-531. doi: 10.1016/0021-8693(89)90315-3.

14. Dauns J., Hofmann K.H. The representation of biregular rings by sheaves // Math. Z. 1966. Vol. 91. P. 103-123. doi: 10.1007/BF01110158.

15. Davey B.A. Sheaf spaces and sheaves of universal algebras // Math. Z. 1973. Vol. 134, no 4, P. 275-290. doi: $10.1007 /$ BF01214692 .

16. Birkhoff G. Lattice theory. Providence, R.I.: Amer. Math. Soc., 1967. 418 p.

17. Миклин А.В., Чермных В.В. O drl-полукольцах // Мат. вестник педвузов и университетов Волго-Вятского региона. 2014. Т. 16. с. 87-95.

Поступила 7.04.2020

После доработки 23.04.2020

Принята к публикации 11.05.2020

Чермных Василий Владимирович

д-р физ.-мат. наук, доцент

главный науч. сотрудник

Сыктывкарский государственный университет им. Питирима Сорокина

г. Сыктывкар

e-mail:vv146@mail.ru

Чермных Оксана Владимировна

канд. физ.-мат. наук

доцент каф. фундаментальной математики

Вятский государственный университет

г. Киров

usr00458@vyatsu.ru

\section{REFERENCES}

1. Rao P.R. Lattice ordered semirings. Math. Sem. Notes, Kobe Univ., 1981, vol. 9, pp. 119-149.

2. Swamy K.L.N. Dually residuated lattice ordered semigroups. Math. Ann., 1965, vol. 159, pp. 105-114. doi: 10.1007 BF01360284.

3. Chermnykh O.V. On drl-semigroups and drl-semirings. Chebyshevskii Sb., 2016, vol. 17, no. 4, pp. 167-179 (in Russian). doi: 10.22405/2226-8383-2016-17-4-167-179.

4. Chermnykh V.V., Chermnykh O.V. Functional representations of lattice-ordered semirings. Siberian Electronic Mathematical Reports, 2017, vol. 14, pp. 946-971 (in Russian). doi: $10.17377 /$ semi.2017.14.080 .

5. Chermnykh O.V. Functional representations of lattice-ordered semirings. II. Siberian Electronic Mathematical Reports, 2018, vol. 15, pp. 677-684 (in Russian). doi: 10.17377/semi.2018.15.053 .

6. Pierce R.S. Modules over commutative regular rings. Mem. Amer. Math. Soc., 1967, vol. 70, pp. 1-112. doi: $10.1090 / \mathrm{memo} / 0070$.

7. Koh K. On a representation of strongly harmonic ring by sheaves. Pacif. J. Math., 1972, vol. 41, no. 2, pp. 459-468. doi: 10.2140/pjm.1972.41.459.

8. Mulvey C.J. A generalization of Gelfand duality. J. Algebra, 1979, vol. 56, no. 2, pp. 499-505. doi: 10.1016/0021-8693(79)90352-1.

9. Filipoiu A. Compact sheaves of lattices and normal lattices. Math. Jap., 1991, vol. 36, no. 2, pp. 381-386.

10. Chermnykh V.V. Representation of positive semirings by sections. Russian Math. Surveys, 1992, vol. 47, no. 5, pp. 180-182. doi: 10.1070/RM1992v047n05ABEH000948.

11. Chermnykh V.V. Functional representations of semirings. J. Math. Sci., 2012, vol. 187, no 2, pp. 187-267. doi: 10.1007/s10958-012-1062-2 .

12. Mulvey C.J. Compact ringed spaces. J. Algebra, 1978, vol. 52, no. 2, pp. 411-436. doi: 10.1016/00218693(78)90248-X.

13. Simmons H. Compact representations - the lattice theory of compact ringed spaces. J. Algebra, 1989, vol. 126, no. 2, pp. 493-531. doi: 10.1016/0021-8693(89)90315-3 . 
14. Dauns J., Hofmann K.H. The representation of biregular rings by sheaves. Math. Z., 1966, vol. 91, pp. 103-123. doi: 10.1007/BF01110158.

15. Davey B.A. Sheaf spaces and sheaves of universal algebras. Math. Z., 1973, vol. 134, no. 4, pp. $275-290$. doi: 10.1007/BF01214692 .

16. Birkhoff G. Lattice Theory. Providence, R.I.: Amer. Math. Soc., 1967, 418 p. ISBN: 0-8218-1025-1.

17. Miklin A.V., Chermnykh V.V., On drl-semirings. Matematicheskiy vestnik pedvuzov $i$ universitetov Volgo-Vyatskogo regiona, 2014, vol. 16, pp. 87-95 (in Russian).

Received April 7, 2020

Revised April 23, 2020

Accepted May 11, 2020

Vasiliy Vladimirovich Chermnykh, Dr. Phys.-Math. Sci., Pitirim Sorokin Syktyvkar State University, Syktyvkar, 167001 Russia, e-mail: vv146@mail.ru .

Oksana Vladimirovna Chermnykh, Cand. Sci. (Phis.-Math.), Vyatka State University, Kirov, 610000 Russia, e-mail: usr00458@vyatsu.ru.

V.V. Chermnykh, O. V. Chermnykh. Functional representations of lattice-ordered semirings. III, Trudy Instituta Matematiki i Mekhaniki URO RAN, 2020, vol. 26, no. 3, pp. 235-248. 